\title{
La Recherche d'une Valeur Ajoutée Cachée dans un Projet de Génie Civil
}

\author{
Bertrand Côté, ing., M.Sc.A. \\ Professeur agrégé \\ Département de génie civil, Université de Sherbrooke \\ Bertrand.Cote@Usherbrooke.ca
}

\begin{abstract}
Résumé
Cet article suggère que dans chaque projet de conception en génie civil se cache une valeur ajoutée cachée qu'il appartient au concepteur de découvrir. La valeur ajoutée proviendra de travaux additionnels qu'on inclura à l'appel d'offres, travaux dont l'ampleur est mineure par rapport aux coûts totaux $d u$ projet, mais dont l'impact est assez important qu'il leur confère un caractère incontournable. L'article propose aux concepteurs une approche simple permettant de rechercher une valeur ajoutée dans n'importe quel type de projet, avec plusieurs exemples en appui.
\end{abstract}

\begin{abstract}
This paper suggests that in any civil engineering design project lies a hidden added value that must be discovered by the designer. The added value will come from additional items that will be included in the call for tenders, items whose magnitude remains small compared to the total costs of the project, but whose impact is such that the items will become a must in the eyes of the client. The paper proposes a simple approach to help design engineers discover a hidden added value in any given type of project, with multiple examples.
\end{abstract}

\section{Introduction}

Tout le processus de conception en génie civil repose sur la satisfaction des besoins du donneur d'ouvrage (client). Mais le processus commence inévitablement en début de mandat par des besoins exprimés. Ceux-ci peuvent être explicites et référer à une série d'ouvrages précis à construire ou ils peuvent simplement être reliés à une performance attendue. Pour compliquer les choses, les besoins exprimés ne correspondent peut- être pas entièrement aux besoins réels attendus et l'ingénieur concepteur doit s'exercer à décoder tous les besoins du client.

Cet article propose une approche novatrice basée sur la recherche d'une valeur ajoutée cachée (VAC). Celle-ci doit rehausser significativement la valeur du produit livrable aux yeux du donneur d'ouvrage et ce, sans augmentation sensible du coût total du projet. Cette valeur ajoutée provient souvent de l'ajout d'un ouvrage précis qui ne correspond pas a priori à un besoin, mais, lorsqu'il est bien identifié, devient incontournable aux yeux du client, au même titre que l'ensemble des ouvrages découlant de besoins réels.

La démarche proposée ici peut être appliquée systématiquement en fin de période de préparation des plans et devis. Elle permet au concepteur de livrer au client un ouvrage qui répond à ses besoins et qui les surpasse même.

\section{Approche d'identification}

Au fur et à mesure que les plans et devis progressent, apparaissent de nombreux éléments sous forme de cotes aux plans, de coûts au bordereau d'estimation, d'aires de circulation pour piétons sur les plans, etc. Un concepteur qui adhère à une pratique rigoureuse de la recherche de la valeur ajoutée cachée questionnera cet ensemble d'éléments :

A- L'ouvrage conçu peut-il devenir un repère unique dans le paysage ?

Vers la fin des années soixante, l'ingénieur André Donaldson a conçu le premier réservoir d'eau potable surélevé de la municipalité de Rock Forest. En révisant ses plans, il se rend compte que le niveau d'eau dans le réservoir, lorsque plein, atteint presque la cote de 1000 pieds. Les plans finals ont été modifiés pour instaurer la cote de 1000 pieds. Ce réservoir demeure un repère 
visuel d'élévation important dans le paysage des environs de Sherbrooke.

B- Un record quelconque se cache-t-il dans l'ouvrage conçu?

En 1989, l'ingénieur Pierre Blais a conçu la passerelle piétonnière suspendue du Parc de la Gorge de la municipalité de Coaticook (figure 1).

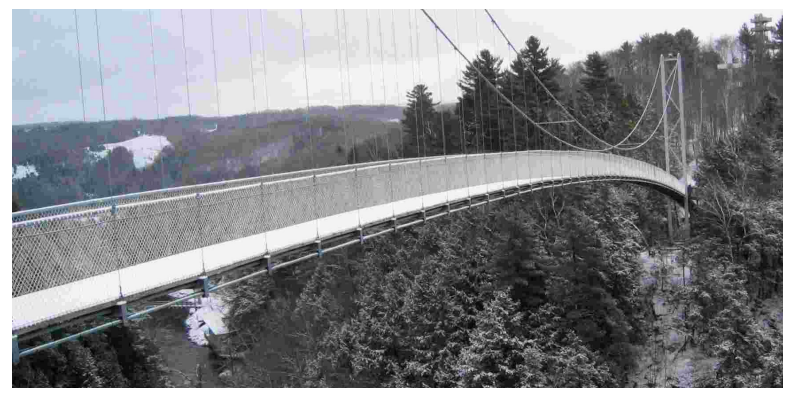

Figure 1. Pont de la Gorge de Coaticook

En révisant ses plans, il s'étonne de la longueur de la portée de l'ouvrage et se demande si d'autres ouvrages ailleurs dans le monde rivalisent avec le sien. Après vérification, il réalise qu'en augmentant légèrement la portée, la passerelle devenait la plus longue du genre au monde. Cette modification a mérité à la ville de Coaticook un certificat de record du monde Guinness et est une grande source de fierté pour Monsieur le maire André Langevin.

C- Le patrimoine du secteur où est construit l'ouvrage peut-il être davantage mis en valeur ? Pensez à l'affichage, à l'éclairage, etc. Un volet artistique peut-il être intégré à l'ouvrage conçu ?

En 1996, l'ingénieur Bertrand Côté a été mandaté pour préparer les plans et devis des travaux de réfection d'un tronçon de la rue Merry Nord dans la ville de Magog. Cette réfection couvrait non seulement la chaussée et les trottoirs, mais également les infrastructures : aqueduc, égouts domestiques et égouts pluviaux. Le tronçon visé par les travaux se situe devant Le Vieux Clocher de Magog, un endroit où des artistes de tout genre se produisent depuis 1975. L'ingénieur Côté eu l'idée du Trottoir des artistes et de modifier les plans du trottoir en face du théâtre pour placer dans le béton des plaques de granit sur lesquelles sont inscrits, année par année, les noms des principaux artistes qui sont montés sur les planches du Vieux Clocher. Près d'une centaine de noms, de Félix Leclerc à Diane Dufresne, en passant par Shawn Phillips et le Stephen Barry Blues Band, ont été gravés dans la série de dalles de granit disposées entre la rue Principale et l'entrée du Vieux Clocher. L'inscription de la figure 2 apparaît sur la première pierre. Le texte, rédigé par l'ingénieur concepteur, fait allusion au mont
Orford, qui surplombe la ville de Magog, au poète bien connu Alfred Desrochers, ainsi qu'au granit des villes frontalières.

Ce trottoir a valu à la ville de Magog une couverture médiatique soutenue pendant des semaines et a fait la

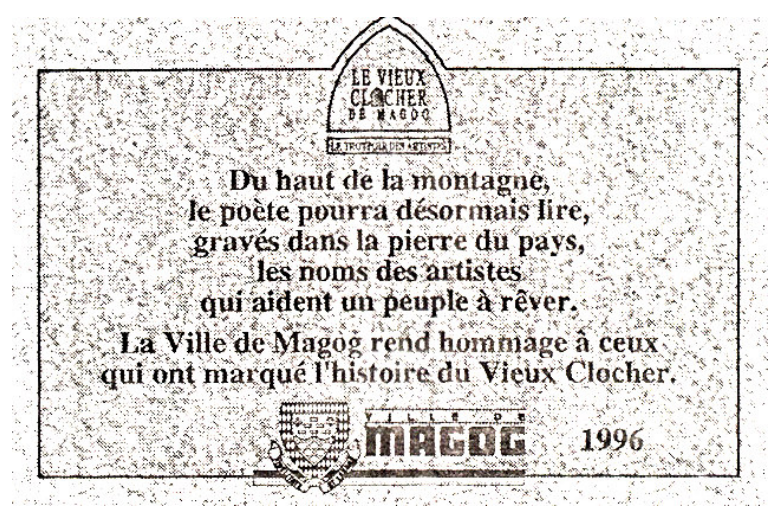

Figure 2. Première Dalle de Granit

joie de Monsieur le maire Denis Lacasse. Ce trottoir peut être apprécié par tous ceux qui font la file à l'extérieur du Vieux Clocher, en l'absence de neige et de glace, bien entendu. L'ajout périodique de plaques a aussi été prévu dans le concept original. Le Trottoir des artistes a coûté moins de 4000 \$ à l'intérieur de travaux totalisant $900000 \$$.

D- La présence de l'ouvrage permet-elle d'exploiter un volet éducatif?

Une étude sommaire portant sur l'enlèvement des ruines d'un ancien barrage situé en amont d'un pont dans la municipalité de Stanstead a conclu qu'il n'y avait aucun avantage à réaliser les travaux. $\mathrm{Au}$ contraire, ceux-ci auraient pu causer préjudice à l'environnement. L'étude a plutôt recommandé d'exploiter la présence des ruines et de créer, pour le bénéfice des piétons empruntant le pont, une plaquette historique éducative montrant une photo du barrage original, accompagnée d'un texte décrivant l'industrie qui exploitait le barrage.

E- La faune peut-elle bénéficier de la présence de l'ouvrage ? Cette question est citée en exemple pour illustrer que tout est permis dans la recherche de la VAC.

Plusieurs travaux d'aménagement extérieurs, ou même de construction en pleine ville, pourraient être adaptés afin de favoriser la nidification des oiseaux. Des aires humides pourraient également être facilement prévues dans nombre de travaux d'aménagement extérieurs.

F- La règle d'or: observez et posez-vous des questions. Laissez l'artiste qui sommeille en vous apparaître. Osez. 


\section{3. Étude de cas}

En 1995, la ville de Magog décide de régler le conflit de circulation qui existait entre piétons et cyclistes sur le pont Merry en construisant une passerelle piétonnière parallèlement au pont. L'ingénieur municipal Charles Fournier et l'ingénieur consultant Bertrand Côté en tracent les grandes lignes sur l'endos d'un napperon lors d'un dîner d'affaires dans un restaurant de Magog. Ils décidèrent, dès ce moment, que le côté de la passerelle faisant face au lac devrait être doté d'une arche joignant les deux rives, par souci d'esthétique et d'allègement visuel de la structure (figures 3 et 4).

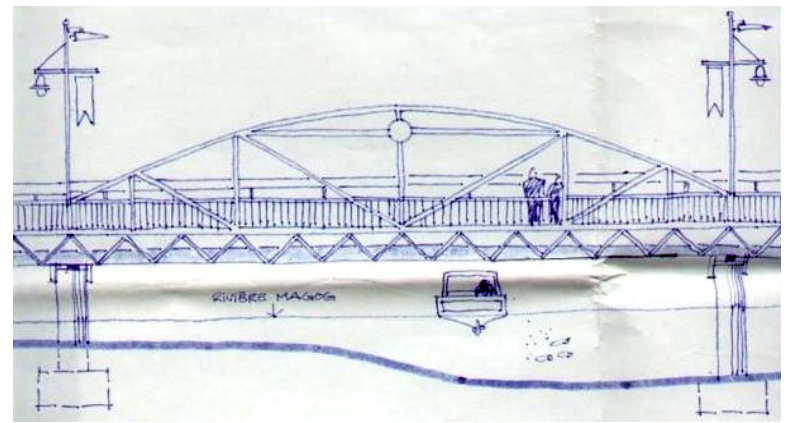

Figure 3. Croquis Stylisé de la Passerelle

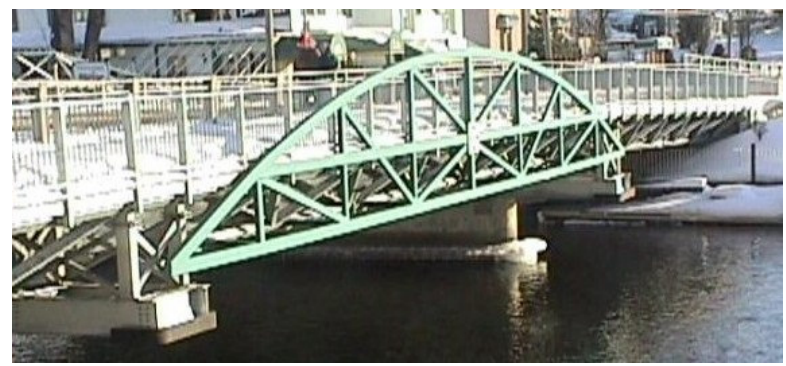

Figure 4. Photographie de la Passerelle

L'arche faite d'aluminium devait, à l'origine, être peinte complètement en vert, incluant rayons et segments. L'ingénieur Bertrand Côté décida alors de laisser le carreau du point focal des segments de l'arche à sa couleur métallique naturelle, sans trop de raison (figure 4). À noter sur la figure 3 que ce carreau était à l'origine un cercle.

Ce n'est que lorsqu'on lui demanda de préparer un texte pour la cérémonie d'inauguration officielle de la passerelle, que la valeur ajoutée de ce détail lui apparut :

«Les aspects techniques et géométriques de l'arche décorative de la passerelle évoquent plusieurs caractéristiques de la ville de Magog et de ses citoyens. L'arche et la passerelle sont faites d'acier galvanisé par un procédé qui requiert de l'électricité, rappelant que Magog est propriétaire de son réseau d'électricité.

La plaque centrale est le point focal des segments qui y convergent, rappelant que Magog est la ville-centre de la MRC de Memphrémagog. La plaque n'est pas recouverte de peinture et révèle l'aspect véritable de l'acier galvanisé, à l'instar de la sincérité des résidants. Elle regroupe quatre boulons géométriquement disposés pour suggérer les quatre points cardinaux, d'o'u proviennent les nombreux visiteurs qui viennent à chaque année admirer la beauté des paysages de la région.

Le périmètre de l'arche est constitué de seulement deux éléments : une corde à la base, qui évoque la droiture des bâtisseurs de la Ville, et un arc qui fait le pont entre les deux rives de la rivière Magog peuplées depuis longtemps par les citoyens de Magog.

On retrouve également, dans la disposition des segments de la structure, la forme d'une croix, symbole de la protection perpétuelle souhaitée à tous ceux qui emprunteront ce nouveau passage...ou qui navigueront en dessous.

Finalement, la couleur verte annonce l'attachement acharné des gens de la région à leur environnement, au grand bénéfice de tous ceux et celles qui savent en profiter. »

La valeur ajoutée du projet dans ce cas était bel et bien le texte rédigé par le concepteur, lequel découlait d'un examen minutieux de la géométrie de l'ouvrage et exploitait un détail de peinture qui avait été décidé inconsciemment. À noter que ce détail avait un coût nul voire négatif, si l'on tient compte de l'économie de peinture qui en a découlé.

\section{Conclusion}

La recherche de la valeur ajoutée cachée pourrait se faire à l'intérieur d'une démarche planifiée vers la fin de la préparation des plans et devis. Elle permet souvent de conférer une valeur collective au projet, qui va au-delà des besoins réels du client. Dans un contexte de réalisation de travaux de génie civil, où des sommes d'argent considérables sont consacrées à des ouvrages dont la visibilité est nulle parce qu'ils sont enfouis, elle permet souvent de rehausser l'image corporative du client. Le tout à peu, et parfois même, sans, frais.

\section{Remerciements}

L'auteur tient à remercier M. Charles Fournier, ing., directeur du développement, des travaux publics et des services techniques de la ville de Magog ainsi que $\mathbf{M}$. Wilfrid Morin, ing., président du Groupe Teknika. 